\title{
Respecting and Protecting the Right of Minor Sexual Autonomy
}

\author{
Kang Li \\ Law School, Fujian Normal University, Fuzhou, 350108, China
}

\begin{abstract}
The right of sexual autonomy is not only a natural problem, but also a humanistic problem. In the process of transformation from "biological man" to "rational man", minors with certain rationality enjoy certain sexual autonomy. The basic concept of juvenile sexual autonomy includes respecting sexual freedom, safeguarding sexual dignity and promoting sexual autonomy. The sexual autonomy of minors faces two basic paths: tolerance and sternness; Has two functions: defense and attack. The state needs to fulfill the minimum obligation of respect and provide the space for the minor's voluntary autonomy. On this basis, the state should also fulfill the duty of protection to protect the space of minors' autonomy from being infringed.
\end{abstract}

Keywords: Minors, Sexual freedom, Sexual autonomy, State obligation.

\section{Introduction}

After the promulgation of China's Civil Code, the revision of the Law on the Protection for Minors has also come to an end. There will be new reforms in the protection for minors, but there is still not much progress of the area of minors' spiritual sexual rights. The society is always concerned about the material rights of minors to be sexually assaulted, but does not or dare not to pay attention to the spiritual rights of minors. Because we are still in the era of "Talking about sex can make your face darker", the spiritual rights of adults can not be guaranteed, let alone minors as a special main body.

With the development of social pluralism, the traditional sexual concept gradually fad, and sexual rights gradually entered the research field of civil law and constitution. Especially in practice, the demands of sexual rights of special groups are increasing gradually. Minor as the most special subject of sexual rights, material rights are greatly protected, but spiritual rights are ignored. The most typical spiritual sexual right is to decide and choose one's own sexual behavior and way. This paper argues that minors also to enjoy this right, which can be defined as sexual autonomy.

\section{The Basic Idea of Sexual Autonomy for Minors}

\subsection{Respecting the Sexual Freedom of Minors}

Freedom is a kind of basic need, basic desire and basic purpose of human beings[1]. Minors also have their own sexual desire, which is the basic condition for the realization of minors' self-awareness. The determination of sexual autonomy is the embodiment of the independent relationship between minors and others. Sexual autonomy is not only the freedom from the natural field, but also the freedom from the social field. As the independent people and the owner of the natural attributes, minors entered the society and produce certain social attribute. To understand and recognize the sexual autonomy of minors is to respect and clarify the sexual freedom of minors.

\subsection{Guaranteeing the Sexual Dignity of Minors}

In civil law, the sexual autonomy of minors is the personality interests they enjoy based on the sexual elements in their personality. Sexual autonomy in human rights is a necessary sexual benefit of minors to change from "biological person" to "social person". This right has the nature of basic human rights and is the minor can not be infringed on the right of personality. Therefore, the determination of the sexual autonomy of minors is to answer the sexual dignity of minors from the perspective of personality rights.

\subsection{Carrying Forward the Sexual Autonomy of Minors}

Sex is a biological concept. It also is a social concept. Only people have the fundamental right to engage in sexual activity as they wish[2]. It is precisely because man to elevate the animal instinct for the spiritual demand that he can give play to his autonomous ability. They can determinate the sexual autonomy of minors, in essence, which is to carry forward the sexual autonomy of minors, so that minors can decide their own sexual behavior and sexual mode independently at some time, so as to meet their own needs. "The only sex that is not true to nature is sex that cannot be performed."[3] It is permissible under minors to pursue any kind of sexual pleasure as long as it does not violate the law, public order or good custom, and does not interfere with the rights of others. Of course, the minor here refers to the minor with complete autonomous ability.

\section{The Operation Path of Minors' Sexual Autonomy}

Sexual rights are the existence of practicality, and it is through this practicality that law plays its role. In modern society, the legal recognition or prohibition on the sexual autonomy of minors directly determines their sexual fate. The criminal law focuses on the protection for the sexual autonomy of minors, emphasizing that the sexual autonomy of minors must not be violated. While the civil law protects the sexual autonomy of minors, it also pays attention to their sexual dignity. The law of modern society under the rule of law is based on rights, caring for human nature, respecting consent, opposing violence, tolerating pluralism, and striving to realize sexual freedom, independence and liberation, which is the development course and basic trend of modern social 
behavior legislation[4]. The operation of minors' sexual autonomy is also experiencing the basic path of tolerance and sternness.

\subsection{The Law Tolerantly Protects the Sexual Autonomy of Minors}

People can do all the actions that are not prohibited by law. In China, there is no law prohibiting minors' sexual autonomy The State and society should accept the equal right of minors and adults to sexual autonomy. Along with the further opening of sexual thinking, some countries have recognized the sexual rights of minorities. Spain, for example, has included the cost of sex reassignment surgery in its national welfare, and has confirmed that people of the same sex do not need to undergo sex reassignment surgery in order to obtain legal transgender status. At first, the mainstream often thought that psychosexual people suffer from diseases, and under the activities of sexual openness, psychosexual people have been recognized to have their own rights of sexual autonomy. Minors can express their sexual desires, put forward their own sexual needs, choose their own sexual partners, and refuse forced sex while performing sexual autonomy. All these is the rights that minors should enjoy as human beings.

Of course, the Constitution seeks for substantive equality, that is, minors are less mature than adults in terms of sexual maturity and cognitive ability, and the law needs to make up for this gap to protect the sexual rights of minors. For minors, the law is tolerant, but restricted by traditional sexual morality, people are afraid to explore the sexual rights of minors. Reproduction-centered sex keeps people quiet about their sexual rights. Pleasure-centered sex makes people love and dislike their partners. Instead, we today put sex in people's relationships. Minors may have their own relationships to manage and may choose their own sexual partners for that reason. The law takes an indifferent attitude, which in itself is tolerance.

\subsection{The Law Severely Punishes Violations of Minors' Sexual Autonomy}

Because of the particularity of minors, their sexual autonomy is more vulnerable to external harm. The law shows a harsh side of those who violate his human will. In this respect, the severity of the law is increasing and the scope of its severity is expanding. In order to severely punish the behavior of rapping young girls, the most severe contact theory was used in the identification of the behavior. In compulsory indecency, it is the recognition and guarantee of the sexual autonomy of minors with incomplete sexual autonomy to include the children with incomplete sexual autonomy into the scope of objects. The Law on the Protection for Minors also makes it clear that parents or guardians have no right to exercise voluntary autonomy on behalf of minors.

Even some countries have certain restrictions on minors that violate their sexual autonomy. The juvenile delinquent act of Canada, for example, provides that sexually immoral children should be sent to industrial schools or juvenile correctional institutions in accordance with federal or provincial regulations. All these indicate that in terms of sexual rights, minors enjoy closer protection, and the state is more severe in punishing the behavior of infringing minors' sexual autonomy.

\section{The Function of Minors' Sexual Autonomy}

Minors can decide their own sexual life and sexual style, express their own sexual desire, and choose their own sexual partners. The essence of the sexual autonomy of minors is the right of freedom, which manifests the freedom of the will of minors. Minors can make appropriate choices of their own interests and realize their sexual rights and obligations independently. The right of freedom from modern society ruled by law includes two dimensions: positive freedom and negative freedom. Positive freedom requires minors to take the initiative to exercise the right of freedom, while negative freedom requires the state and society to protect the rights of minors by means of omission. In the field of juvenile sexual autonomy, two functions are extended simultaneously, namely, defense function and attack function.

\subsection{The Defensive Function of the Sexual Autonomy of Minors}

Negative freedom is the premise of the exercise in freedom. Without right to defense, freedom will not exist [5]. The right of defense is the basis and premise of freedom. Its concept explains the function of the autonomy under the negative freedom well. The right of defense was first put forward by Germany. Its function is to guarantee individual freedom from the interference with public power, so as to create the free space of people. The sexual autonomy of minors belongs to the category of freedom of minors in essence, and its defense object is the state. The right of defense is not a specific constitutional right, but constitutional right. From the perspective of the sexual autonomy of minors, the state needs to fulfill the negative obligation to omission in order to ensure that minors have enough free space for their sexual rights. As long as the state does not act, the right to defence is effective.

\subsection{The Assault Function of the Sexual Autonomy of Minors}

Positive freedom is the key to the exercise in freedom. The existence of positive freedom is the real connotation and goal of freedom. The sexual autonomy of minors is not only infringed by the rights of the state, but also influenced by parents and society. Due to the physical immaturity of minors, the degree of rationality is not enough, so in the exercise of their sexual autonomy, will inevitably be subject to some passive restrictions, may be directly related to the physical and mental health of minors. This requires the minors to take the initiative in the act of sexual autonomy, and the state therefore needs to provide conditions for the minors to act of sexual autonomy.

\section{State Obligations in the Sexual Autonomy of Minors}

From the perspective of the relationship between individual and state, the connotation of constitutional rights is determined by the relationship between individual and state. The constitution respects and guarantees the human dignity of 
every human being. The sexual autonomy of minors is a special form of personal dignity. It is necessary for the state to respect and protect the sexual autonomy of minors. This is also decided by the two functions of the minor sexual autonomy.

\subsection{The State Shall Assume the Obligation to Respect the Sexual Autonomy of Minors}

In the system of state obligations, the primary obligation that the state should undertake is "respecting". The state needs to give certain free space to the sexual autonomy of minors, and shall not interfere, interfere or infringe on them at will. China's traditional sexual morality and ethics put people in an environment of sexual repression. Even in a free and democratic modern society, sex is still a topic that many people do not want to talk about. However, due to the privacy of sex itself, and the fact that sex is scattered in the daily life of members of society, it is impossible to control all people's sexual behavior even with strict orders, social networks and legal norms[6]. So the state tries to use morality and custom, and even science, to regulate people's sexuality. For example, in Britain at the end of the 19th century, psychiatrists and psychologists considered unmarried pregnancy to be a sign of mental retardation.

Sexual instinct is the essence of natural person. The difference between sex and other instincts lies in that sex serves itself and its purpose is itself. As a result, the state's sexual repression of natural persons will make them extremely painful. So you need to liberate sexual freedom, liberate sexual desire. Liberating sexual freedom does not mean indulging sexual freedom; Liberating the libido does not mean indulging the lust. Sexual rights require a tolerant social environment, which is what the negative obligation to the State to do nothing is all about.

The State respects the material rights of minors. However, in the aspect of spiritual sexual rights, especially the sexual autonomy of minors, the state does not respect the degree enough. Although the state takes a laissez-faire attitude to the sexual autonomy of minors, it has used a variety of methods to restrict the sexual autonomy of minors, for example, high schools prohibit minors from falling in love. Minors can not decide their own sexual behavior or sexual way, dare not to choose their own sexual partners, do not want to express their own sexual desire. Therefore, let the sexual physiological maturity of minors go before the sexual psychological maturity, produced a lot of sexual ignorance, sexual disorder, sexual diseases, so that the minors are more painful. On this basis, it extends the distorted sexual culture of yellow culture. This culture injects minors into thinking that sex is just making love and that sex is just an expression of desire.

Minors, like adults, have their own sexual needs and desires. The state's repression will only lead underage sex astray. Therefore, the state needs to respect the sexual autonomy of minors through various forms. The legislature has the duty to enact laws that shall not infringe on the sexual autonomy of minors. Administrative organs have the obligation not to directly restrict the sexual autonomy of minors when the law has not provided for it. Judicial organs need to respect the sexual autonomy of minors in interpreting laws and making judgments.

\subsection{The State Shall Undertake the Obligation to Protect the Sexual Autonomy of Minors}

The sexual autonomy of minors requires not only the minimum respect, but also the protection for higher dimensions. Adults appear to be at their ease of exercising sexual autonomy. In the absence of a deep understanding of love and sex, minors may behave in ways that are not understood by outsiders. The state has an obligation to protect the space for minors to engage in sexual activity.

When minors take voluntary autonomy, the first thing they encounter is the obstruction of their parents or guardians. Parents generally believe that the sex of minors is evil and that minors are not sexually mature. It essentially confuses sexual physiological maturity with sexual psychological maturity. Moreover, parental nurturing and care are the first gate of sexual cognition for minors. Bad family relations, or discordant relationship between parents will produce the result that minors' sexual concepts or sexual behavior will produce some alienation. Secondly, school sex education is not enough. In today's exam-oriented education, students can only receive education about examinations in school, but there is no curriculum about sex education. Even if there were biology lessons, the teachers kept their mouths shut. This creates sexual ignorance of minors, which leads to bad sexual problems. Moreover, the news media and society are biased against the sexual autonomy of minors. In some rape cases, it is often reported in the media that underage clothing is the biggest cause. Minors' freedom of dressing has become the reason for sexual assault, which is the prejudice against minors' sexual autonomy.

In order to avoid the alienation of the sexual autonomy of minors, the state is required to care for minors and realize their sexual autonomy by active protection. The main body of national protection obligation is the state, which is mainly shared by the legislative, administrative and judicial organs.

\subsubsection{Legislative Protection Obligation}

The sexual autonomy of minors has been the essence of constitutional rights. Criminal law, civil law and administrative law all protect the sexual autonomy of minors to a certain extent. However, the sexual autonomy of minors is still not clearly recognized. The confirmation and protection for special legislation is the main basis and guarantee for minors to exercise their sexual autonomy. In the Australian Capital Territory, people over the age of 16 can have sex with each other, whether of the opposite sex or not, as long as they are consensual and not close family members[7]. In the United Kingdom, the Gillick case extended the right of children to self-determination, that is, if the child has enough understanding and intelligence to make his own decision on the issue that needs to be decided, then his parental rights should be compromised on the right of the child to make his own decision[8].

China does not affirm the sexual autonomy of minors, but is somewhat coy about it. The Law on the Protection for Minors 
elaborates on the protection content of family, school, society and other subjects, ignoring the self-protection of minors. In the area of sexual autonomy for minors, self-protection is the most needed. This is the essence of the sexual autonomy of minors. The legislature should consider the type, subject and scope of the sexual autonomy of minors, as well as the relationship with the family, society and school, so as to clarify the exercise and protection for the sexual autonomy of minors.

\subsubsection{Administrative Protection Obligation}

The behavior of administrative agencies regarding the sexual autonomy of minors in accordance with laws and regulations is essentially an act of law enforcement and does not disrespect the sexual autonomy of minors. Only when the administrative organs exercise their functions and powers within the scope of their discretion or formulate administrative laws and regulations should they attach importance to the particularity of the sexual autonomy of minors. In 2018, two male students in a school raped a female student. The administrative detention decision was posted on the school Post Bar and circulated among the students the next day. Although the female student's name and other specific circumstances were not directly stated, the female student had been known by most people in the school in the subsequent treatment. What is left to think about is that after others infringing on the sexual autonomy of minors, administrative agencies should adopt stricter protections, that is to say, the disclosure of minors' identity information must be protected. For administrative agencies, all links must be kept confidential.

\subsubsection{Judicial Protection Obligation}

In China's legal environment, judicial protection has become an important part of the protection for minors' rights. First of all, the provisions of the Constitution, such as "respecting and safeguarding human rights" and "inviolable human dignity", directly bind the judicial organs. Secondly, in the field of juvenile sexual autonomy, the judicial organs have certain obligations of organization and procedure protection. Finally, the judicial power itself has the function of the last line of defense. When the sexual autonomy of minors is violated by the outside world, they can get relief. At this time, the protection obligation to the judicial organ is to give the entity the relief right, that is, the right to win the lawsuit. When judging cases of sexual autonomy of minors, judicial organs should consider the autonomy and particularity of minors, take the sexual rights of minors as the priority protection interests, and make corresponding discretion.

\section{Acknowledgment}

This paper is supported by the funds: Fujian Environmental Protection Technology Project "Water Environment Regulations of Fujian Province (Revision)", project number: 2013R003. The Laws and Regulations Division of the Education Department of Fujian Province Commissioned Project: "School Power List Research", project number: JYTFWT202102.

\section{References}

[1] Wang Haiming. Justice, Equality, Humanity: Moral Principle System of Social Governance [M]. Peking University Press, pp.146, 2000.

[2] Pan Suiming. Social History of Sex [M]. Henan People's Publishing House, pp.53, 1998.

[3] M. Clerc, The Swarm and the Queen: Towards a Deterministic and Adaptive Particle Swarm Optimization [C]. In Proceedings of the IEEE Congress on Evolutionary Computation (CEC), pp. 1951-1957, 1999.

[4] Alfred Kinsey, Translated by Pan Suiming. Jin Xi Report - Human Male Sexual Behavior [M]. Guangming Daily Press, editor's preface, pp.19, 989.

[5] Li Yongjun. Sexual Rights and Law[M]. Science Press, pp.86, 2009.

[6] Xia Zhenglin. From Fundamental Rights to Constitutional Rights [M]. Law Press, pp.68, 2018.

[7] Malinovsky, Translated by Fei Xiaotong. Cultural Theory [M], Huaxia Publishing House, pp.48, 2002.

[8] Sun Yunxiao \& Zhang Meiying. Contemporary Minor Legal Translation Collection, Australia Volume [M], China Procuratorial Publishing House, pp.4, 2006.

[9] Sun Yunxiao \& Zhang Meiying. Contemporary Minor Law Translation Collection, British Volume, China Procuratorial Press, pp.6, 2005.

\section{Author Profile}

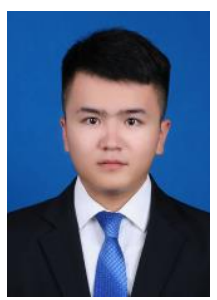

Kang Li (1997-), male, is a Master Degree candidate at the Law School, Fujian Normal University, and has been studying for a Master's degree since 2019. 